East African Medical Journal Vol. 84 No. 1 January 2007

CAESAREAN SECTION: INTRA-OPERATIVE BLOOD LOSS AND ITS RESTITUTION

A.F. Faponle, FWACS, FMCA, Consultant Anaesthetist/Senior Lecturer in Anaesthesia, Department of Anaesthesia and O.N. Makinde, FWACS, Senior Lecturer \Consultant Obstetrician and Gynaecologist, Obafemi Awolowo University Teaching Hospitals Complex, Mle-Ife, Nigeria

Request for reprints to: Dr. A.F. Faponle, Department of Anaesthesia, Obafemi Awolowo University, Ile-Ife, Nigeria

\title{
CAESAREAN SECTION: INTRA-OPERATIVE BLOOD LOSS AND ITS RESTITUTION
}

\author{
A.F. FAPONLE and O.N. MAKINDE
}

\begin{abstract}
Objectives: To determine the rate of blood loss and its restitution during Caesarean section and make recommendations to improve our practice.

Design: Prospective study.

Setting: Obafemi Awolowo University Teaching Hospitals Complex, Ile-Ife, Nigeria from 1st January 2005 to December 2005.

Subjects: All term Caesarean sections performed during the period.

Results: Six hundred and forty one patients met the inclusion criteria. The mean maternal age was 30.42 years \pm 5.5 (Range 16-44 years). Fifty seven patients $(8.9 \%)$ were transfused. Major blood loss $(>1000 \mathrm{ml})$ was reported in $7.6 \%$ of the patients. The main causes of major blood loss during surgery were placental disorders and pre-eclampsia. The main indications for transfusion were placental disorders, pre-eclampsia and breech presentation. The packed cell volume, status of the anaesthetist and the ASA grading were other factors, which had statistical association with blood transfusion. The risk of major blood loss and transfusion were negligible in patients presenting for foetal distress, cephalo-pelvic disproportion and breech presentation.

Conclusion: The rate of blood transfusion during Caesarian section is high. Antepartum haemorrhage and pre-eclampsia were predictable indications for major blood loss and transfusion. Major blood loss and transfusion rate were negligible in patients presenting with foetal distress, cephalo-pelvic disproportion and breech presentation.
\end{abstract}

\section{INTRODUCTION}

Caesarean section is the most common major surgical procedure carried out in most hospitals. The incidence of Caesarean section in these hospitals has continued to rise in most high risk obstetric units. Sudden haemorrhage may occur with any manipulation of the highly vascular term uterus. Thus blood transfusion may be necessary for this operation. Provision of adequate blood for obstetrics often continues to challenge transfusion services in most hospitals. This is also known to delay surgery as often donors are not available or patients cannot pay for cross-matching. The protocol in most hospitals is for two units of blood to be provided before Caesarian section. Often, most patients are not transfused intra-operatively but the surgery would have been delayed because of the need to cross-match blood for surgery. Thus, if patients at high risk for transfusion can be identified, routine cross-match will be mandatory for such. The delays in surgical intervention and the significant cost of blood transfusion may be eliminated.

In developed countries, through better education, blood transfusion during elective Caesarean section is now so infrequent that routine cross-match is not recommended (1). This is yet to be achieved in many developing countries.

The aim of this study was to determine the rate of blood loss and its restitution during Caesarean section in a tertiary hospital in Nigeria. 


\section{MATERIALS AND METHODS}

This was a prospective study of all term ( $\geq 37$ weeks) Caesarean sections performed between $1^{\text {st }}$ January and December $31^{\text {st }} 2005$ at the Obafemi Awolowo University Teaching Hospitals Complex (OAUTHC), Ile-Ife. Data collected included demographic, American Society of Anaesthesiologists (ASA) grading, coexisting conditions, presence of clinical anaemia, indication for Caesarean section, preoperative packed cell volume, surgical details including the type of incision, location of the placenta, number of pints of blood cross-matched for surgery, and number of pints transfused intra-operatively, intra-operative blood loss and post-operative packed cell volume. Descriptive statistics was used for analysis. Categorical variables was analysed using Chi-square analysis. Logistic regression analysis was used to determine the interaction between risk factors and transfusion outcomes.

\section{RESULTS}

There were 641 Caesarean sections during the period. The mean maternal age was 30.42 years \pm 5.5 (Range 16-44 years). The mean pre-operative PCV was $33.04 \pm 5.66 \%$ (18-48\%). The indications for Caesarean section are as shown in Table 1.

Fifty seven patients $(8.9 \%)$ were transfused intra-operatively and within 24 hours of surgery. Table 2 shows the transfusion pattern for each of the indications for surgery.

Table 1

Indications for Caesarean sections

\begin{tabular}{lrr}
\hline Indication & No. & $(\%)$ \\
\hline Prolonged labour & 50 & 7.8 \\
Failure to progress in labour & 106 & 16.5 \\
Foetal distress & 80 & 12.5 \\
Placenta praevia & 27 & 4.2 \\
Pre-eclampsia/eclampsia & 60 & 9.4 \\
Repeat Caesarean sections & 135 & 21.1 \\
Post-term & 66 & 10.3 \\
Breech & 19 & 3.0 \\
Obstructed labour & 32 & 5.0 \\
APH & 21 & 3.3 \\
CPD & 21 & 3.3 \\
Others & 24 & 3.7 \\
\hline Total & 641 & 100 \\
\hline
\end{tabular}

Table 2

Indication for Caesarean section and transfusion

\begin{tabular}{lrrrr}
\hline Indication & \multicolumn{2}{c}{ Transfused } & \multicolumn{2}{c}{$\begin{array}{c}\text { Not } \\
\text { transfused }\end{array}$} \\
& No. & $(\%)$ & No. & $(\%)$ \\
\hline Prolonged labour & 2 & 4.0 & 48 & 96.0 \\
Failure to progress & 5 & 4.7 & 101 & 95.3 \\
Foetal distress & 1 & 1.2 & 79 & 98.8 \\
Placenta praevia & 7 & 25.9 & 20 & 74.1 \\
Pre-eclampsia & 6 & 10.0 & 54 & 90.0 \\
Repeat section & 9 & 6.7 & 126 & 93.3 \\
Post-term & 4 & 6.1 & 62 & 93.9 \\
Breech presentation & 2 & 10.5 & 17 & 89.5 \\
Obstructed labour & 3 & 9.4 & 29 & 90.6 \\
APH & 12 & 57.1 & 9 & 42.9 \\
CPD & 1 & 4.8 & 20 & 95.2 \\
Others & 5 & 26.3 & 19 & 73.7 \\
\hline Total & 57 & & 584 & 91.1 \\
\hline
\end{tabular}

The duration of surgery did not have a statistically significant influence on blood transfusion though the mean duration of surgery was higher in patients who were transfused $(55.47 \pm 34.55$ min vs $49.46 \pm$ $25.93 \mathrm{~min}$ ). Transfused patients had lower packed cell volume compared with those who were not ( $29.61 \pm 4.88$ vs $33.66 \pm 3.77$ ). All the patients who were transfused were given general anaesthesia. None of the 22 patients given spinal anaesthesia was transfused.

The mean number of units cross-matched for patients who were transfused was higher compared with those who were not ( $2.33 \pm 0.7$ vs 1.59 units \pm $0.62)$. This was statistically significant. Two patients died within 24 hours of surgery giving a mortality of $0.3 \%$.

A logistic regression analysis revealed that prolonged labour $(\mathrm{OR}=1.4$ ) previous Caesarean section (OR 1.1), breech presentation ( $O R=3.3$ ), APH $(O R=9.9$ ) were significant indications for blood transfusion during Caesarean section. Also factors aśsociated with blood transfusion included abnormal placenta location $(\mathrm{OR}=3.95)$ and blood loss greater than $I L(O R=1.9)$ during Caesarean section.

A higher proportion of patients with abnormal placenta had blood transfusion (17.2\% vs $9.1 \%$ ) and this was statistically significant $(p<0.05)$. The surgeons status had no impact on blood transfusion during Caesarean section though operations carried out by residents alone recorded a higher ratio of 
Table 3

Indication for Caesarean section and blood loss

\begin{tabular}{lrrrrr}
\hline & \multicolumn{3}{c}{ Blood loss } & & \\
Indication & $<1000 \mathrm{ml}$ & \multicolumn{1}{c}{$>$} & Total \\
& No. & $(\%)$ & No. & $(\%)$ & \\
\hline Prolonged labour & 42 & 93.3 & 3 & 6.7 & 45 \\
Failure to progress & 96 & 94.1 & 6 & 5.9 & 102 \\
Foetal distress & 77 & 96.3 & 3 & 3.8 & 80 \\
Placenta praevia & 17 & 65.4 & 9 & 34.6 & 26 \\
Pre-eclampsia & 53 & 91.4 & 5 & 18.6 & 58 \\
Repeat Caesarean section & 125 & 95.4 & 6 & 4.6 & 131 \\
Post-term & 55 & 91.7 & 5 & 8.3 & 60 \\
Breech presentation & 18 & 94.7 & 1 & 5.3 & 19 \\
Obstructed labour & 30 & 93.8 & 2 & 6.3 & 32 \\
APH & 18 & 85.7 & 3 & 14.3 & 21 \\
CPD & 21 & 100 & 0 & 0 & 21 \\
Others & 20 & 83.3 & 4 & 16.7 & 24 \\
\hline Total & 572 & 92.4 & 47 & 7.6 & 619 \\
\hline
\end{tabular}

Note: Blood loss in 22 patients was not recorded

blood transfusion. Also, where a nurse was the main anaesthetist, more patients were transfused $(\mathrm{P}<0.01)$.

The ASA status is also statistically suggestive of an association with, blood transfusion. The higher the ASA, the more likely the patient will be transfused $(P<0.001)$. Age had no influence on blood transfusion. The mean ages of the transfused and non-transfused patients were similar $(30.56 \pm$ 5.74 vs $30.41 \pm 5.53$ ).

\section{DISCUSSION}

This study has investigated transfusion practice in 641 women subjected to lower segment Caesarean section. The overall percentage of transfused patients was high $(8.9 \%)$ compared with reports from developed countries where rates as low as $0.66 \%-1.1 \%$ have been reported $(1,2)$. This rate compares favourably with that of a study in Malawi $(7.2 \%)$ (3) but is much lower than what was found in Ethiopia (19\%) (4). A similar study in another tertiary hospital in Nigeria reported a transfusion rate of $5.6 \%$ (5).

Major blood loss (>IL) was reported in $7.6 \%$ of the patients. The emerging trend in this study suggests risk of transfusion and major blood loss is negligible in patients presenting for foetal distress, cephalopelvic disproportion and breech presentation. Placenta abnormalities and pre- eclampsia carried the expected increased transfusion and major blood loss risk. In a similar study, patients presenting for elective repeat or breech Caesarean section were found to have a negligible risk of blood transfusion and major blood loss (6). In a study by Magann and others, women who had non-elective Caesarean section were noted to have higher postpartum haemorrhage than those delivered electively (7). Patients with placenta praevia have been documented as having a transfusion rate 6.5 times higher than in control subjects (8).

In most hospitals in developing countries blood and blood products are scarce mainly because of the increasing demand of blood for surgery associated with declining supply. The supply of blood is declining because of difficulties in finding suitable donors. With such scenario, it is important that blood use is done rationally, so as to maximize the benefits of blood transfusion for those in whom its use is considered necessary.

Blood availability has been shown to be a significant factor in maternal mortality. Usually, the increased blood volume associated with normal pregnancy typically accommodates the obligatory blood loss that occurs during vaginal or Caesarean delivery. However in some patients, blood loss may overwhelm compensatory mechanism and may result in hypovolemia and shock with a significant threat for both mother and foetus. Despite advances in prevention, diagnosis and treatment, massive blood 
loss during pregnancy still remains a threat. The development of better knowledge of tissue oxygenation and the fear of transmitting infectious diseases are some of the factors that have led anaesthesiologists to transfuse blood only when required.

The protocol for surgery in our hospital is that two pints of blood should be made available before Caesarean section. Thus surgery is often delayed while suitable blood donors are being sought or while blood is being cross-matched. If the blood is not used during surgery, the operation would have been delayed unnecessarily. This may also have an impact on the foetal outcome as well as maternal morbidity and mortality.

Transfused patients had a higher number of units cross-matched for surgery in this study. This may suggest that on clinical grounds alone, clinicians may be able to predict which patients would require blood transfusion during surgery. Such factors such as the placenta location, the ASA status, the status of the anaesthetist, pre-operative haematocrit which were found to be statistical correlates to blood transfusion during surgery in this study are factors which surgeons could use to make clinical decisions.

Although much of the information is dated, there is a substantial body of evidence indicating that transfusion practice, varies among physicians and institutions (9). Many authors concluded that differences in transfusion practice suggest inappropriate use. From various reports, there appears to be an association between pre-operative haemoglobin concentration, intra-operative estimated blood loss and post-operative mortality $(10,11)$.

The mean pre-operative haematocrit in these patients was low. Transfused patients had an even lower haematocrit than those who were not. This suggests that patients with lower haematocrit are likely to be transfused during Caesarean section. This is usually so in clinical practice. The higher level of pre-operative haematocrit which is seen in patients of higher socioeconomic status may account for the lower intra-operative transfusion seen in developed countries. Anaemia per se is not an indication for Caesarean section but evidently it is an indication for blood transfusion during Caesarean section.

Two patients died within 24 hours of surgery in this study. One patient had disseminated intravascular coagulation following placenta praevia and died following transfusion of ten pints of blood intra and post operatively. Another patient died following valvular complications of rheumatic heart disease during Caesarean section.

Large numbers are required to make definitive statements about ability to reduce the need for screened and cross-matched blood. However, using this methodology it is possible to provide this data. A multi-hospital study of transfusion practice in obstetrics which will involve large number of palients will be required to provide this data. This may guide clinicians in making requests for screened cross-matched blood before Caesarean section. In view of emerging infections like the pandemic HIV and hepatitis $B$ viral infections, recourse to blood transfusion during Caesarean section should only be when and only when needful. This may be the time to review the protocol for blood availability for Caesarean section.

\section{REFERENCES}

1. Deballi P., Spalin T. and Muir H.A. Are routine type and screen order necessary for Caesarean section? Anuesthesiology. 2002; 96: Suppl 1.

2. Imberti R., Preseglio I., Trotta V., et al. Blood transfusion during Caesarean section. A 12 years retrospective analysis. Acta. Anaesthesioligica Belgium. 1990; 41: 139-144.

3. Fenton P.M. Blood transfusion for Caesarean section in Malawi. A study of requirements, amount given and effect on mortality. Anaesthesia. 1999; 54: 1055-1058.

4. Tadesse E., Adane M. and Abiyou M. Caeserean section deliveries at Tikur Anbessa Teaching Hospital, Ethiopia. East Afr. Med. J. 1996; 73: 619-622.

5. Imaregiaye C.O. and Ande A.B. Risk factors for blood transfusion during $\mathrm{C}$-section in a tertiary hospital. Med. Sci. Manag. 2006; 12: 269-272.

6. Ransom S.B., Fundaro G. and Dombrowski M.P. Cost effectiveness of routine blood type and screen testing for Caesarean section. J. Reprod. Med. 1999; 44: 592-594.

7. Magann F.F., Lanneau G. and Morrison J.C. Post partum haemorrhage after delivery:an analysis of risk factors. Southern Med. J. 2005; 98: 681-685.

8. Takayama T., Minakami H., Koke T., et al. Risks associated with Caesarean section in women with placenta praevia. J. Obstet. Gynaecol. Research. 1999; 23: 375-379.

9. Herbert P.C., McDonald P.J. and Tinmouth A. Overview of transfusion practices in peri-operative and critical care. Canadian f. Anesthesia. 2003; 50: 565-S75.

10. Carson J.L., Spence R.K. and Poses R.M. Severity of anaemia and operative mortality and morbidity. Lancet. 1988; 1: 727-729.

11. Spence R.K., Carson J.A., Poses R., et al. Elective surgery without transfusion: influence of pre-operative haemoglobin level and blood loss on mortality. Amer. J. Surg. 1990; 159: 320-324. 